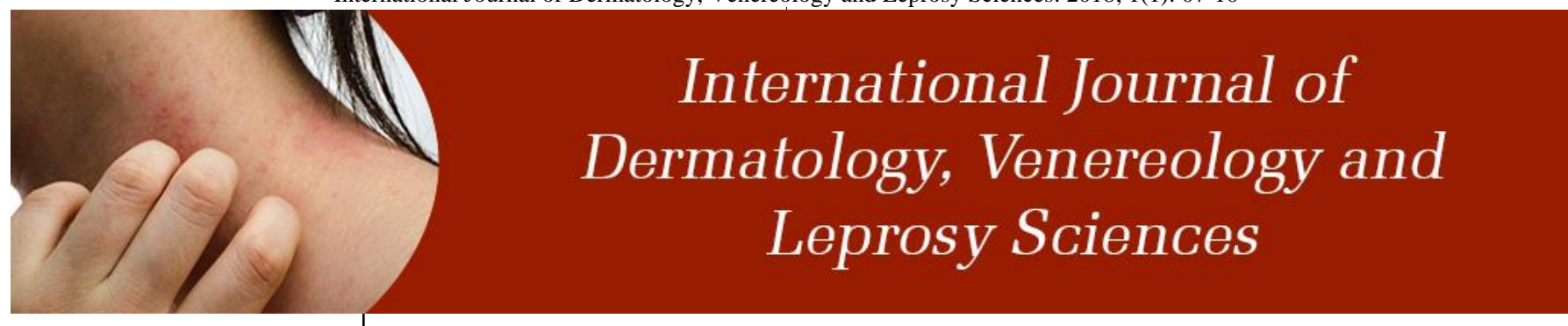

E-ISSN: 2664-942X

P-ISSN: 2664-9411

www.dermatologypaper.com/

Derma 2018; 1(1): 07-10

Received: 07-11-2017

Accepted: 10-12-2017

Dr. Aaryan Joshi

Department of Dermatology,

Nobel Medical College

Teaching Hospital,

Biratnagar, Nepal
Corresponding Author:

Dr. Aaryan Joshi

Department of Dermatology,

Nobel Medical College

Teaching Hospital,

Biratnagar, Nepal

\title{
Assessment of cases of Vitiligo reported to department: A clinical study
}

\section{Dr. Aaryan Joshi}

DOI: $\underline{\text { https://doi.org/10.33545/26649411.2018.v1.i1a.3 }}$

\begin{abstract}
Background: Vitiligo is known to occur with many autoimmune diseases. The present study was conducted to assess cases of Vitiligo in study group.

Materials \& Methods: The present study was conducted on 112 cases of vitiligo of both genders. The extent of body surface area involvement was measured.

Results: Out of 112 participants, males were 52 and females were 60 . The site of involvement was upper limb in 14, lower limb in 8, lips in 4, scalp, face \& neck I 52, trunck in 23 and eyelid in 11 . The difference was significant $(P<0.05)$. Most common triggering factors are identified by patients $(40)$, physical trauma (48), surgery (3), psychological stress (12), pregnancy (5) and medical illness (4). The difference was significant $(P<0.05)$. Segmental pattern was seen in 14 , non- segmental in 68 , mixed in 20 , vitiligo universalis in 5 , genital involvement in 3 and mucosal involvement in 2 .

Conclusion: Vitiligo is a common skin disease. Most cases were in females and Scalp, face, neck were the mostly affected.
\end{abstract}

Keywords: Face, Scalp, Vitiligo

\section{Introduction}

Vitiligo is known to occur with many autoimmune diseases, and there are many reports of its association with Type 1 diabetes mellitus unlike its association with Type 2 diabetes mellitus. Few studies show the association of vitiligo with Type 2 diabetes. Vitiligo is an acquired, noncontagious disorder, in which progressive, patchy loss of pigmentation of skin and often overlying hair, and mucous membranes, results from the loss of melanocytes from the involved areas ${ }^{[1]}$.

The exact etiology of vitiligo is poorly understood and is often considered as a multifactorial disease with a complex pathogenesis encompassing several postulations implicating autoimmune, cytotoxic, biochemical, oxidant- antioxidant, viral, and neural mechanisms for destruction of the melanocyte function in genetically predisposed. The presence of autoimmune diseases like autoimmune thyroiditis, Grave's disease, Addison's disease, diabetes mellitus, alopecia areata is predominant factors ${ }^{[2]}$.

Autoimmune mechanisms are thought to be responsible in the pathogenesis of vitiligo (especially in generalised or focal nondermatomal vitiligo). This is supported by an increased incidence of antibodies found in people with vitiligo ${ }^{[3]}$. Furthermore, vitiligo is often associated with autoimmune diseases, such as thyroid diseases, pernicious anaemia, and diabetes mellitus. Another indication that vitiligo may be due to an autoimmune mechanism is that melanocyte antibodies have been found in people with vitiligo, and their incidence correlates with disease activity ${ }^{[4]}$. Involvement of cellular immunity has been considered because $\mathrm{T}$ lymphocytes and macrophages in perilesional skin have also been frequently reported. Regarding segmental vitiligo, the neural hypothesis suggests that it is due to an accumulation of a neurochemical substance which decreases melanin production ${ }^{[5]}$.

Some dermatological outpatient records show the incidence of vitiligo to be $3 \%$ to $4 \%$ in India although an incidence as high as $8.8 \%$ has also been reported. In the Indian states of Gujarat and Rajasthan, the prevalence of vitiligo is very high being described by some as of epidemic proportions ${ }^{[6]}$. The present study was conducted to assess cases of Vitiligo in study group.

\section{Materials and methods}

The present study was conducted in the department of Dermatology. It comprised of 112 
cases of vitiligo of both genders. The study was approved from institutional ethical committee. All participants were informed regarding the study and written consent was obtained.

Information such as name, age, gender etc. was recorded. In all patients, presence of other cutaneous and systemic disorders was recorded. The extent of body surface area involvement was measured by Wallace rule of nine and various clinical patterns were classified according to Vitiligo Global Issues Consensus Conference 2011-12 report. Results thus obtained were subjected to statistical analysis. $\mathrm{P}$ value less than 0.05 was considered significant.

\section{Results}

Table I: Distribution of participants

\begin{tabular}{|c|c|c|}
\hline & Total- 112 & \\
\hline Gender & Males & Females \\
\hline Number & 52 & 60 \\
\hline
\end{tabular}

Table I shows that out of 112 participants, males were 52 and females were 60

Table II: Site of occurrence

\begin{tabular}{|c|c|c|}
\hline Site & Number & P value \\
\hline Upper limb & 14 \\
\hline Lower limb & 8 \\
\hline Lips & 4 \\
\hline Scalp, face, neck & 52 \\
\hline Trunk & 23 & \\
\hline Eyelid & 11 & \\
\hline
\end{tabular}

Table II, graph I shows that site of involvement was upper limb in 14 , lower limb in 8 , lips in 4 , scalp, face \& neck I
52 , trunck in 23 and eyelid in 11 . The difference was significant $(P<0.05)$.

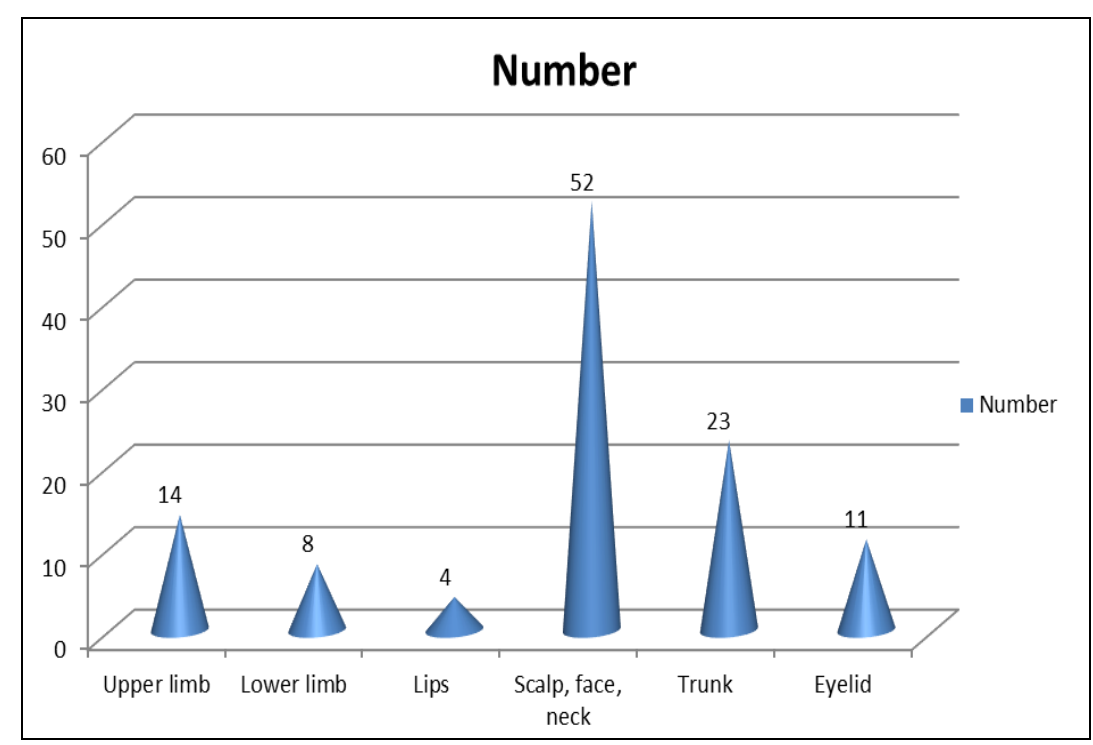

Graph I: Site of occurrence

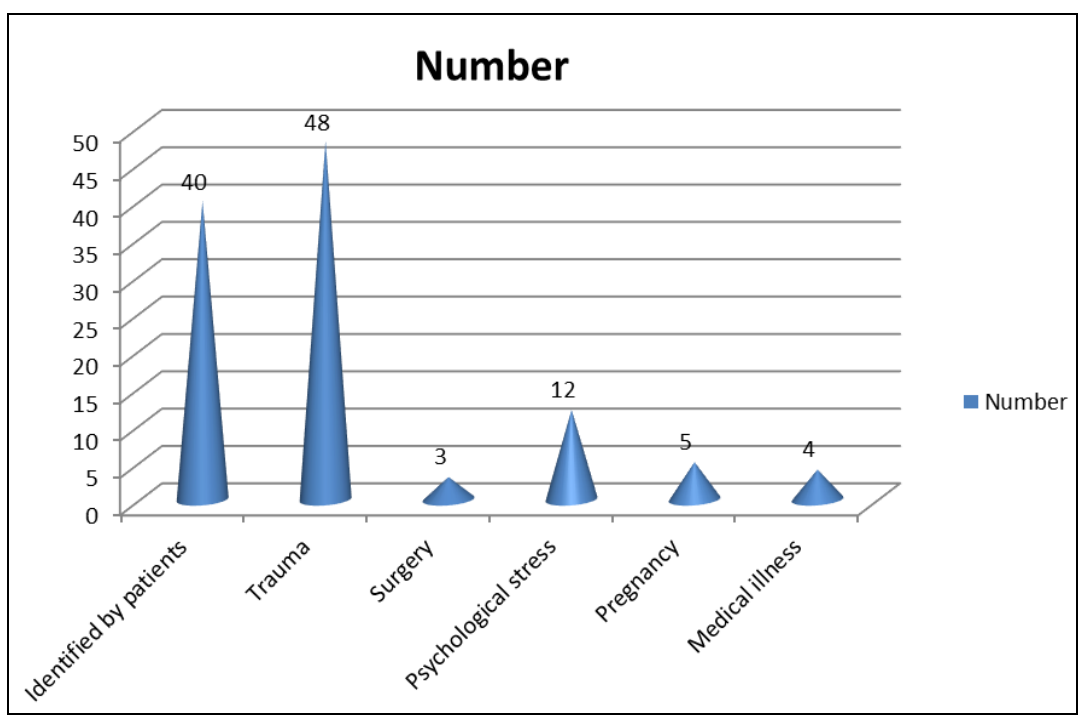

Graph II: Triggering factors 
Graph II shows that most common triggering factors are identified by patients (40), physical trauma (48), surgery (3), psychological stress (12), pregnancy (5) and medical illness (4). The difference was significant $(P<0.05)$.

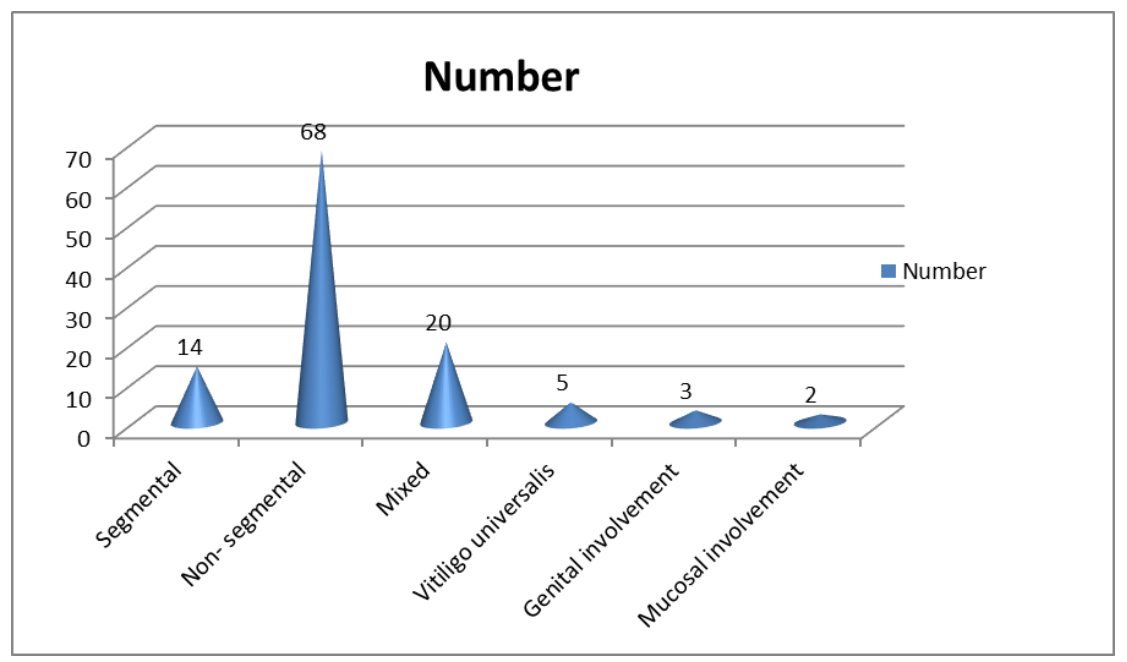

Graph III: Clinical pattern

Graph III shows that segmental pattern was seen in 14 , nonsegmental in 68 , mixed in 20 , vitiligo universalis in 5 , genital involvement in 3 and mucosal involvement in 2 .

\section{Discussion}

Factors such as poor nutrition, emotional stress, autoimmunity, trauma, drugs, infections, sepsis, and exposure to the sun, chemicals, and toxins are often considered to trigger it. Clinically, the usual age of onset is before 20 years of age in nearly half of the cases. It affects both genders equally at any age but most studies report a peak incidence between 18 and 21 years (mean 24 years) ${ }^{[7]}$. Vitiligo is not life threatening and is mostly asymptomatic, although it does increase the risk of sunburn of the affected areas. The association of vitiligo and skin cancer remains an area of controversy. The occurrence of skin cancer in longlasting vitiligo is rare, although studies have demonstrated increased PUVA associated skin cancers. ${ }^{8}$ The effects of vitiligo can be both cosmetically and psychologically devastating, resulting in low self-esteem and poor body image. The anxieties regarding the disease occur against a background of a lack of understanding of the etiology and unpredictability of the course ${ }^{[9]}$. The present study was conducted to assess cases of Vitiligo in study group.

In this study, out of 112 participants, males were 52 and females were 60. The site of involvement was upper limb in 14 , lower limb in 8 , lips in 4 , scalp, face \& neck I 52, trunck in 23 and eyelid in 11 .

Shajil et al. ${ }^{[10]}$ found that incidence of vitiligo among new patients was $1.84 \%$. A total of 365 patients were included in the study, out of which females $(68.4 \%)$ were found to be more affected than males $(31.6 \%)$, in a ratio of $2.1: 1$. The majority $(32.82 \%)$ of the patients was in their second decade of life, and $58.63 \%$ of the patients were unmarried. A positive family history was present in $50(13.7 \%)$ patients. Trauma $(3.84 \%)$ was the most common precipitating factor, followed by emotional upset $(2.19 \%)$ and physical pressure $(0.82 \%)$. History of progressive vitiligo was obtained in $65.59 \%$ of the patients. Vitiligo vulgaris $(64.9 \%)$ was the most common morphological pattern; other patterns seen were focal vitiligo (18.6\%), acrofacial vitiligo (0.8\%), mucosal vitiligo (14.8\%), segmental vitiligo (1.4\%), and universal vitiligo $(8.2 \%)$.

In this study, common triggering factors are identified by patients (40), physical trauma (48), surgery (3), psychological stress (12), pregnancy (5) and medical illness (4). Dave et al. ${ }^{[11]}$ found that there were 449 men and 496 women (m:f 1:1.1) aged between 2 and 83 years (mean 24.4 years) and having vitiligo for 1 week to 64 years (mean 5.1 years). The majority, $478(50.6 \%)$ patients were aged $\leq 20$ years and $248(26.2 \%)$ were children aged $\leq 12$ years. The age at the onset was between 6 months and 82 years (mean 20.5 years), and the majority $674(71.3 \%)$ patients had it before 25 years of age. The consultation time was within 5 years in $692(73.2 \%)$ patients. A family history of vitiligo was present in $150(15.9 \%)$ patients. The majority 871 (92.2\%) patients had involvement of up to $10 \%$ body surface area and vitiligo vulgaris in $562(59.5 \%)$ and focal vitiligo in $117(18.7 \%)$ patients were the most common clinical types. An association with other systemic disorders was in $124(13.1 \%)$ patients and predominately included thyroid abnormalities and diabetes mellitus.

We found that segmental pattern was seen in 14, nonsegmental in 68 , mixed in 20 , vitiligo universalis in 5 , genital involvement in 3 and mucosal involvement in 2 . Although vitiligo may develop anytime in life, the onset in early infancy or old age is uncommon. Genetic factors, as in patients with affected first-degree relatives, too have suggested to influence the age of onset of vitiligo ${ }^{[12]}$.

\section{Conclusion}

Vitiligo is a common skin disease. Most cases were in females and Scalp, face, neck were the mostly affected.

\section{References}

1. Vora RV, Patel BB, Chaudhary AH, Mehta MJ, Pilani AP. A clinical study of vitiligo in a rural set up of Gujarat. Indian J Community Med. 2014; 39:143- 6.

2. Shah H, Mehta A, Astik B. Clinical and sociodemographic study of vitiligo. Indian J Dermatol Venereol Leprol. 2008; 74:701.

3. Fatani MI, Al Sharif SH, Alfif KA, Khan AS, Hussain WA, Banjar AA. The clinical patterns of vitiligo "hospital- based study" in Makkah region, Saudi 
Arabia. J Dermatol Dermatol Surg. 2014; 18:17-21.

4. Howitz J, Brodthagen H, Schwartz M, Thomsen K. Prevalence of vitiligo. Epidemiological survey on the isle of Bornholm, Denmark. Arch Dermatol. 1977; 113:47-52.

5. Shankar DS, Shashikala K, Madala R. Clinical patterns of vitiligo and its associated co morbidities: A prospective controlled cross sectional study in South India. Indian Dermatol Online J. 2012; 3:114-8.

6. Vrijman C, Hosseinpour D, Bakker JG, Wolkerstorfer A, Bos JD, Van Der Veen JP et al. Provoking factors, including chemicals, in Dutch patients with vitiligo. $\mathrm{Br}$ J Dermatol. 2013; 168:1003-11.

7. Van Geel N, Speeckaert R, Taieb A, Picardo M, Böhm $\mathrm{M}$, Gawkrodger DJ et al. Koebner's phenomenon in vitiligo: European position paper. Pigment Cell Melanoma Res. 2011; 24:564- 73.

8. Handa S, Pandhi R, Kaur I. Vitiligo: A retrospective comparative analysis of treatment modalities in 500 patients. J Dermatol. 2001; 28:461-6.

9. Singh N, Gera V. Evaluation of Zinc Levels in Patients with Vitiligo- A Clinical Study. J Adv Med Dent Scie Res. 2019; 7(7):156-158.

10. Shajil EM, Agrawal D, Vagadia K, Marfatia YS, Begum R. Vitiligo: Clinical profiles in Vadodara, Gujarat. Indian J Dermatol. 2006; 51:100-4.

11. Dave S, Thappa DM, Souza MD. Clinical predictors of outcome in vitiligo. Indian J Dermatol Venereol Leprol 2002; 68:323-5.

12. Lu T, Gao T, Wang A, Jin Y, Li Q, Li C. Vitiligo prevalence study in Shaanxi Province, China. Int $\mathrm{J}$ Dermatol. 2007; 46:47-51. 